\title{
Diagnostic and treatment modalities for patients with cervical lymph node metastases of unknown primary site - current status and challenges
}

\author{
Jens Müller von der Grün ${ }^{1}$, Aykut Tahtali ${ }^{2}$, Shahram Ghanaati ${ }^{3}$, Claus Rödel ${ }^{1,4,5}$ and Panagiotis Balermpas ${ }^{1,4,5^{*}}$ (D)
}

\begin{abstract}
Background and Purpose: This review aims to provide a comprehensive overview of the literature and elucidate open questions for future clinical trials concerning diagnostics and treatment modalities for cervical cancer of unknown primary (CUP).

Methods: A literature search for head and neck CUP was performed with focus on diagnostics and therapies as well as molecular markers.

Results: High level evidence on CUP is limited. However, it seems that a consensus exists regarding the optimal diagnostic procedures. The correct implementation of biomarkers for patient stratification and treatment remains unclear. An even greater dispute dominates about the ideal treatment with publications ranging from sole surgery to surgery with postoperative bilateral radiotherapy with inclusion of the mucosa and concomitant chemotherapy.

Conclusions: Cervical CUP represents a very heterogeneous malignant disease. On this account many aspects concerning treatment optimization remain unclear, despite a considerable number of publications in the past. Future research in form of prospective randomized trials is needed in order to better define patient stratification criteria and enable tailored treatment.
\end{abstract}

Keywords: CUP, Cancer of unknown primary, Cervical, Lymph node, Head and neck cancer

\section{Background}

Cancer of unknown primary site (CUP) includes a various group of metastatic diseases whose primary tumor is not detected after clinical examination and extended diagnostic procedures. Reasons therefore may be involution or a slower growth rate at the primary tumor site, due to different genetic alterations in the primary or the metastases [1]. Dependent on the country, CUP represents $2-8 \%$ of the overall malignancies [2] and $3-5 \%$ of all solid tumors [3-5]. The estimated occurrence of CUP in the head and neck (HNCUP) region varies between 3 and $9 \%$, with histological findings of a

\footnotetext{
* Correspondence: panagiotis.balermpas@kgu.de

1Department of Radiation Oncology, University Hospital, Johann Wolfgang

Goethe University, Theodor Stern Kai 7, 60590 Frankfurt, Germany

${ }^{4}$ German Cancer Research Center (DKFZ), Heidelberg, Germany

Full list of author information is available at the end of the article
}

squamous cell malignancy in $53-77 \%$ of the cases [6-8]. The frequency of a subsequent mucosal emergence of the primary site in the head and neck region varies between 4 and $21 \%$ percent in the studies reviewed [9-28]. The most frequently encountered primary symptom is a cervical mass due to enlarged lymph nodes (94\%) [15], mostly located in level 2 (30-50\%), followed by level 1 and $3(10-20 \%)$ and 4 and $5(5-10 \%)$ [2, 15]. Bilateral involvement of the neck is reported in less than $10 \%$ of the cases $[6,8,15,18,19,29,30]$. When node metastases are found in levels 1-3, the primary site is suspected to be in the head and neck region. Upon affliction of the levels $4-5$, the primary tumor most likely is located below the clavicles [31-33]. The time interval between noting the cervical mass and final diagnosis of HNCUP ranges from 2 to 5 months $[6,8,34]$. 
HNCUP patients are predominantly men, aged 55-65 years, showing typical risk factors for head and neck cancer such as tobacco and alcohol abuse [6, 8, 15, 18, $29,35]$. Patients with human papillomavirus (HPV, 90\% HPV-16), detected in lymph-node metastases represent a different and growing population [36] with a median age of at least five years less than HPV-negative patients, less tobacco and alcohol abuse and significant better prognosis [37, 38].

Since no prospective randomised studies are available for HNCUP patients, the therapeutic strategies for HNCUP differ widely and are based on retrospective studies, clinical experience and institutional policy. They range from surgery or (chemo)-radiotherapy alone to surgery plus adjuvant radiotherapy of various extents with or without concomitant chemotherapy [11, 29, 39, 40]. The prognosis for patients with CUP highly depends on the histology and involved region ranges from poor (adenocarcinoma metastatic to bone, brain and/or viscera) to favorable (e.g., squamous cell carcinoma metastatic to neck lymph nodes). The median survival of the poor prognostic group ranges from 7 to 11 months, whereas the survival of the favorable subset is similar to head and neck carcinomas with known primaries (e.g., HNSCC) [2, 41-43]. Here, we provide a comprehensive review of current diagnostic and therapeutic strategies, discuss open questions and challenges in the management of HNCUP patients like (stage dependent) universus multimodality treatment, RT treatment volumes and the need of concomitant chemotherapy and also propose a treatment algorithm.

\section{Diagnostics: what should be considered standard and which are the implications of new molecular markers?} Clinical examination and diagnostic procedures aim at staging the tumor according to the UICC-TNMclassification system. HNCUP is a diagnosis of exclusion; not until after all workup is completed, the classification can be reduced to solely $\mathrm{N}$ and $\mathrm{M}$ defining CUP.

\section{Patient history and examination}

If the patient history reflects excessive use of alcohol and tobacco, the primary site is unlikely to be situated in the nasopharynx, whereas promiscuity and orogenital contact suggest findings within the oropharynx. Also a history of skin lesions of the head and neck can guide the search [44]. The patient usually presents with a painless, unilateral cervical mass. Affliction of the levels 1-3 indicates the primary site to be located in the head and neck region, whereas a mass in levels $4-5$ suggests the primary tumor situated at the lower neck (e.g., thyroid gland) or below the clavicles [31-33] (Fig. 1). Further examination is performed through exploring the head and upper aerodigestive tract using a nasopharyngoscope.

\section{FNAB}

FNAB (Fine-needle aspiration biopsy) of the cervical mass is the first and most commonly used diagnostic procedure, as it is minimal invasive and associated with a negligible risk of spreading the tumor along the needle's path. After routine staining, the diagnostic sensitivity for metastatic neck lymph nodes ranges from 83 to $97 \%$ with a specifity of $91-100 \%$ when performed by an experienced histopathologist [45].

\section{Immunohistochemistry}

Immunohistochemistry (IHC) is an important tool to identify the tissue's origin. General staining identifies cell morphologies and abnormal/malignant cell populations. Afterwards, an initial IHC-panel for broad cancer types including epithelial, melanocytic and lymphoid markers is used. General markers for carcinomas are the cytokeratins, i.e., intermediate filaments specific to epithelium. Markers for lymphomas are CLA - common leukocyte antigen, ALK1 - anaplastic lymphoma kinase, CD30 and CD43. For melanomas there are S-100, HMB45 - antihuman melanosome, and Melan-A [46]. In case of carcinoma, its subtype is evaluated by considering morphological aspects followed by specific antibodies, such as CK5, CK6, CK7 or TTF-1 [46]. The most common tumor types for HNCUP are squamous cell carcinomas SCC and adenocarcinomas.

\section{Imaging}

Available imaging techniques for patients with HNCUP are CT- and MRI- as well as 18F-fluoro2-deoxyglucose positron emission tomography (FDG-PET)-scans (Fig. 1). A quick, inexpensive, procedure with high spatial resolution is the contrast-enhanced CT-scan from the skull base to clavicles, complemented or substituted by a gadolinium contrast-enhanced MRI with superior soft tissue resolution [43]. In case of a cervical lymph node metastasis, the chance for CT, MRI or both to detect the primary site ranges from 9 to $23 \%$ [7, 47-49]. When suspicious findings on imaging are used to guide biopsy, the chance to find the primary tumor rises up to 60\% [50]. For lymph nodes located in levels $4-5$, additional chest/ abdominal/pelvic CT-scans are recommended [51]. FDG-PET is a useful diagnostic tool when standard radiological work-up is completed with negative or inconclusive results and should be performed before any invasive procedures, which possibly hamper the evaluation of the scans due to iatrogenic induced tissue alteration [52-56]. Its capability for tumor detection is down to a size of $\geq 5 \mathrm{~mm}$. Several studies and reviews adressed the additional benefits of FDG-PET for patients with HNCUP (Table 1) [54-56]. The extent of pre-FDG-PET diagnostic workup differs between the studies, so that it becomes difficult to compare the reported sensitivities 


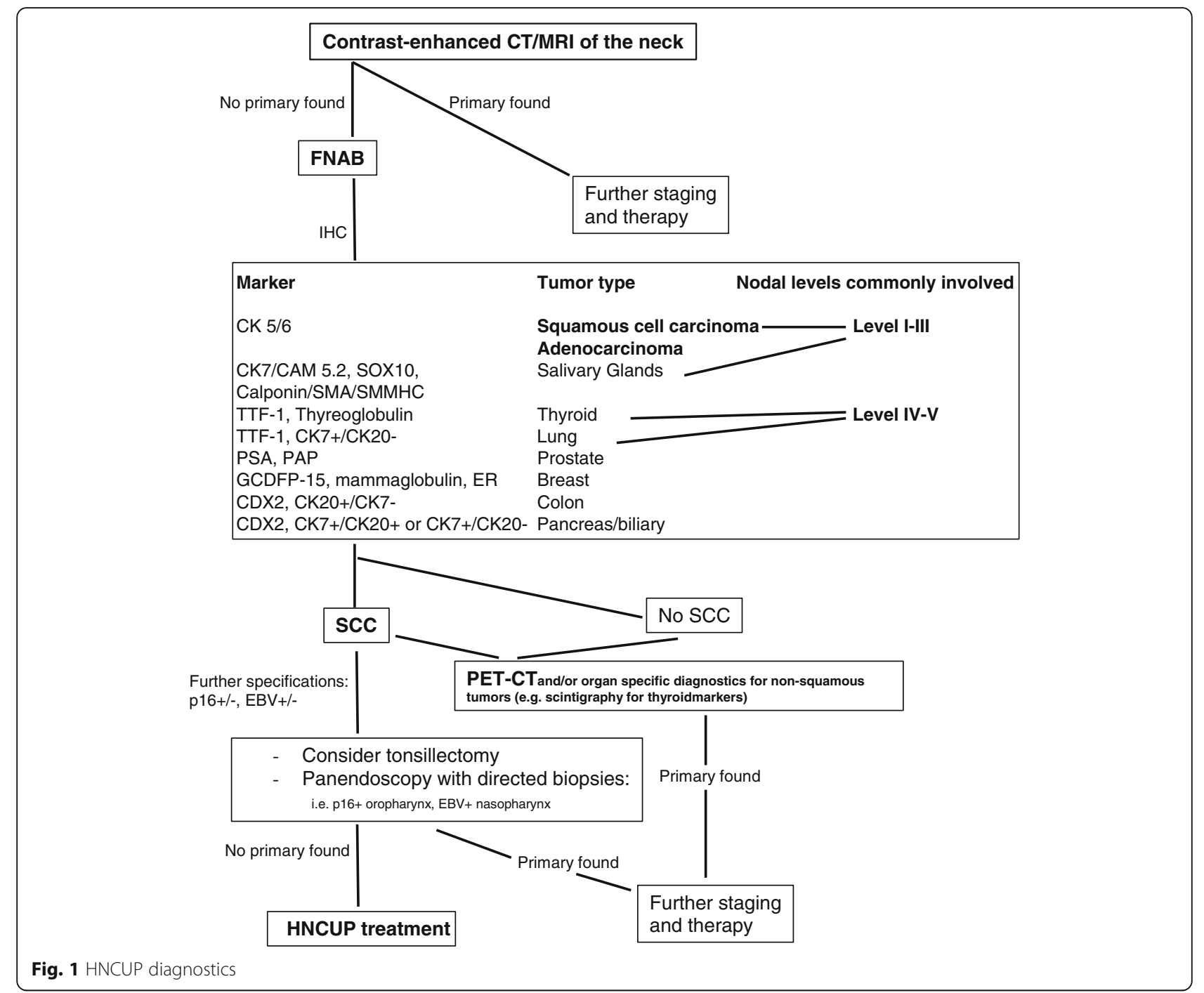

and specifities and to quantify the additional value of PET [57].

\section{Panendoscopy with biopsies}

Panendoscopy of the upper aerodigestive tract (PUADT), including naso-, oro- and hypopharynx as well as laryngoscopy and esophagoscopy, is performed under general anesthesia. Biopsies are taken from radiologically or clinically suspicious sites [43]. Additional bronchoscopy is recommended when indicated by imaging [44]. Repetition of panendoscopy is only indicated when directed biospy failed during the first procedure $[50,58]$. Ipsilateral tonsillectomy leads to primary tumor detection in $18-44.6 \%$ of the cases. Waltonen et al. [47] reported the highest success rate for detection of the primary tumor by PET-CT scans plus panendoscopy with directed biopsies, with or without tonsillectomy (59.6\%).

\section{Molecular studies}

HPV DNA, when found in metastasis, directs the search for the primary tumor to the oropharynx, as the prevalence of HPV in non-oropharyngeal squamous cell cancers currently is only $22 \%$. HPV status can be determined by in-situ hybridization (ISH) or polymerase chain reaction (PCR), dectecting HPV DNA or by HPV E6/E7 RNA expression detected by quantitative reverse transcriptasePCR (qRT-PCR). As a HPV surrogate marker, immunohistochemical staining of p16, a human tumor-suppressor protein [59-65], is also widely used. Despite showing a significantly improved disease-free survival, some authors like Dixon et al. could not find an improved overall survival for p16-positive HNCUP patients in their studies [66]. Other reports showed a significant positive impact of HPV/p16 only when combined with other factors like (non-)smoking [67]. A meta-analysis published in 2007 regarding non-oropharyngeal HNSCC shows congruent results [68]. However, most of the published literature 
Table 1 Reviews on FDG-PET techniques used for patients with HNCUP; NR - not reported; [54-56]

\begin{tabular}{|c|c|c|c|c|c|c|}
\hline Review studies published (Year) & $\begin{array}{l}\text { No. of Studies/ } \\
\text { Patients }\end{array}$ & Technique & $\begin{array}{l}\text { Primary Tumor } \\
\text { Detection Rate (\%) }\end{array}$ & Sensitivity (\%) & Specifity (\%) & $\begin{array}{l}\text { Highest false positive } \\
\text { rate }\end{array}$ \\
\hline $\begin{array}{l}\text { Rusthoven et al., } 2004 \text { [54] } \\
\text { (1994-2003) }\end{array}$ & $16 / 302$ & FDG-PET & 24.5 & 88.3 & 74.9 & Tonsils (39.3\%) \\
\hline $\begin{array}{l}\text { Kwee et al., } 2009 \text { [56] } \\
\text { (2005-2007) }\end{array}$ & $11 / 433$ & FGD-PET/CT & 37 & 84.0 & 84.0 & $\begin{array}{l}\text { Oropharynx (15\%) } \\
\text { Lung (15\%) }\end{array}$ \\
\hline $\begin{array}{l}\text { Al-Ibraheem et al., } 2009 \text { [55] } \\
(2000-2009)\end{array}$ & $8 / 180$ & $\begin{array}{l}\text { FGD-PET } \\
\text { FDG-PET/CT }\end{array}$ & 28.3 & NR & NR & NR overall 16.7\% \\
\hline
\end{tabular}

agrees that $\mathrm{HPV} / \mathrm{p} 16$ is a positive prognostic indicator for HNCUP $[69,70]$.

TP53 (protein name: p53) is a tumor-suppressor gene which is altered in about $50 \%$ of human malignancies, either by mutation or inactivation due to viral or cellular protein interactions leading to p53 degradation [71]. Significantly impaired outcome for patients with mutated p53 status or overexpression of p53 (whose expression directly correlates with the mutated protein, as the second tends to accumulate) in HNCUP and HNSCC has been demonstrated before [72, 73]. Some of the authors also examined the impact of the combined HPV/p53 status on survival and came to the conclusion that p53 could be an independent prognostic factor regardless of the HPV status [74].

Epstein-Barr virus (EBV) is consistently associated with nasopharyngeal carcinoma (NPC), especially with poorly or undifferentiated and nonkeratinizing types [75]. NPC is much more common in southern China and southeast Asia than in Europe or north America [74]. EBVs latent membrane protein 1 is highly suspicious of having a central role in both initiation and progression of the tumor [76-78]. EBV-DNA is routinely detected by PCR with sensitivity and specifity close to 90\% from FNAB samples [79-82].

The data above suggest that the importance of detecting the HPV and EBV-DNA (or their surrogate proteins) in a metastatic lymph node in CUP-disease is high, as it can guide both further diagnostics and treatment (e.g., new directed biopsies or a radiotherapy-boost directed to the assumed primary tumor site) and also predict the patient's outcome. These assays should be implemented in clinical routine for every HNCUP case. Immunohistochemistry for p53 is a simple and inexpensive method for further prognostic stratification and could be used as an additional prognostic parameter.

\section{Therapeutic options}

Due to the lack of randomized trials, the optimal treatment strategies for HNCUP remain controversial. Therapeutic options depend on patient's age, performace status, local extention, the site of the lymph node metastases and their histology. While tumor types other than SCC are often treated likewise cervical metastases with a known primary [83], this review focuses on the treatment of HNSCC-CUP. In former series, the HNCUP treatment aimed for the metastases as well as the suspected primary mucosal site. However, contemporary approaches need to evaluate the benefit of local neck and mucosal control separately, depending on the patient's age and performance status. In early-stage neck disease, monomodal therapy is possible, whereas an advanced-stage neck disease usually requires an aggressive multimodal approach, comparable to locally advanced head and neck cancer [83]. Table 2 summarizes larger studies on HNCUP-therapy, including nodal stages of the patients treated, treatment modalities, radiotherapy and surgery specifications and finally control rates and survival data [9-28].

\section{Is there a need for multimodality treatment for early-stage neck disease?}

Early-stage HNCUP is defined as pN1 or mobile pN2a without extracapsular extention (ECE). Adequate regional control was reported both by studies performing surgery or radiotherapy (RT) as monotherapy. A bias exists, since usually patients with greater neck burden are treated more likely with RT primarily [10]. Although policy-dependend approaches prefer surgery alone with the option of salvage-RT $[16,84]$ or vice versa [15], there is some evidence for primary surgery: only pathology after surgery reliably proves ECE, which then necessitates RT with concomitant chemotherapy (CTx) and the vast majority of the patients in the published series implemented this approach (Table 2). In pN1 or pN2a situations without ECE, postoperative RT has not proven clear benefit regarding locoregional control or survival $[85,86]$. However, some of the few studies addressing this topic are biased, due to their retrospective nature and the simplified statistics used $[87,88]$, so that the role of postoperative RT in these situations remains unclear. However, when RT was postponed and used for salvage treatment only, ultimate control above the clavicles still reached more than $90 \%$ in $\mathrm{pN} 1$ situations without ECE [84]. Surgery should also be followed by adjuvant RT in cases of connective tissue invasion (ECE), more than one involved node and a likelihood of residual 


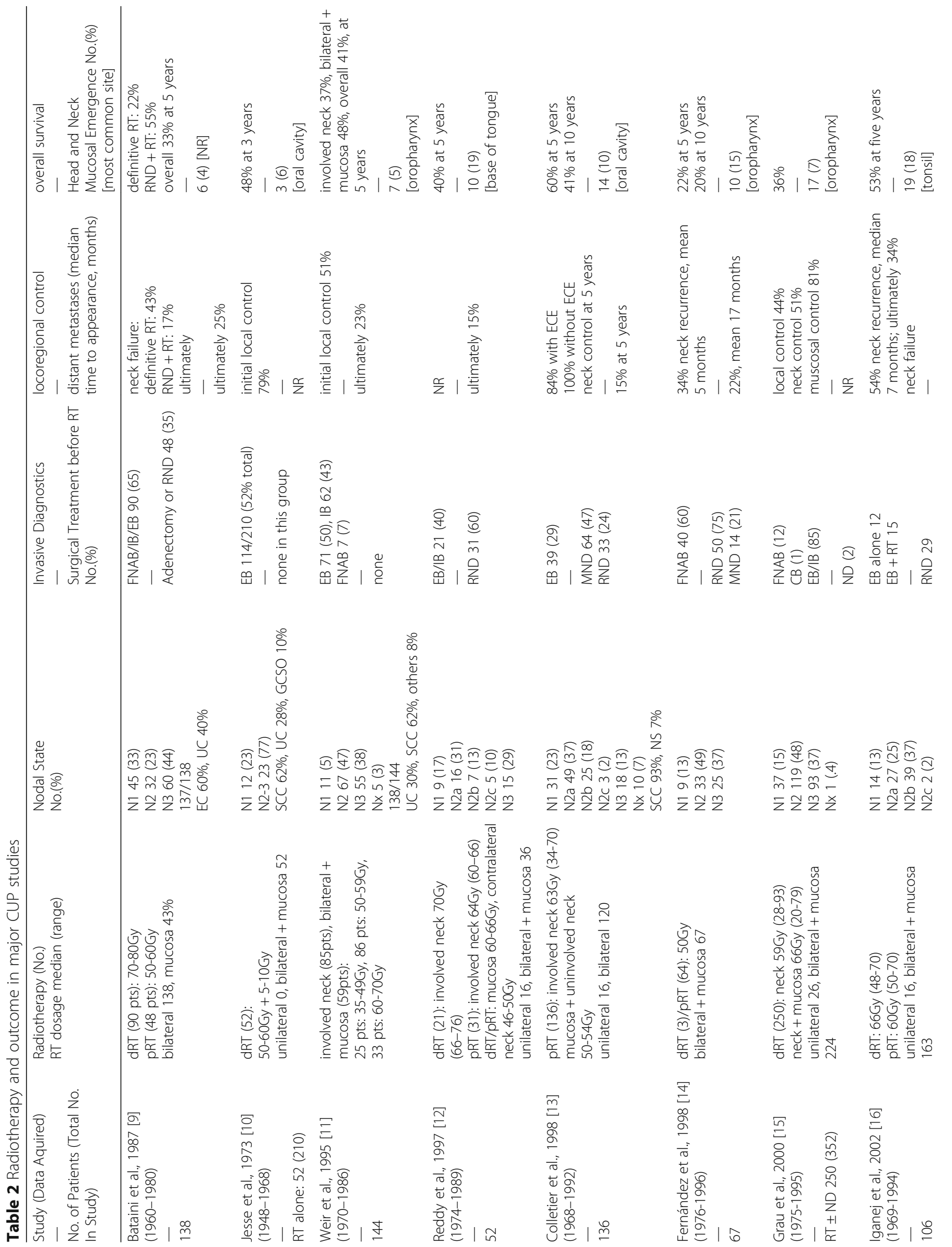




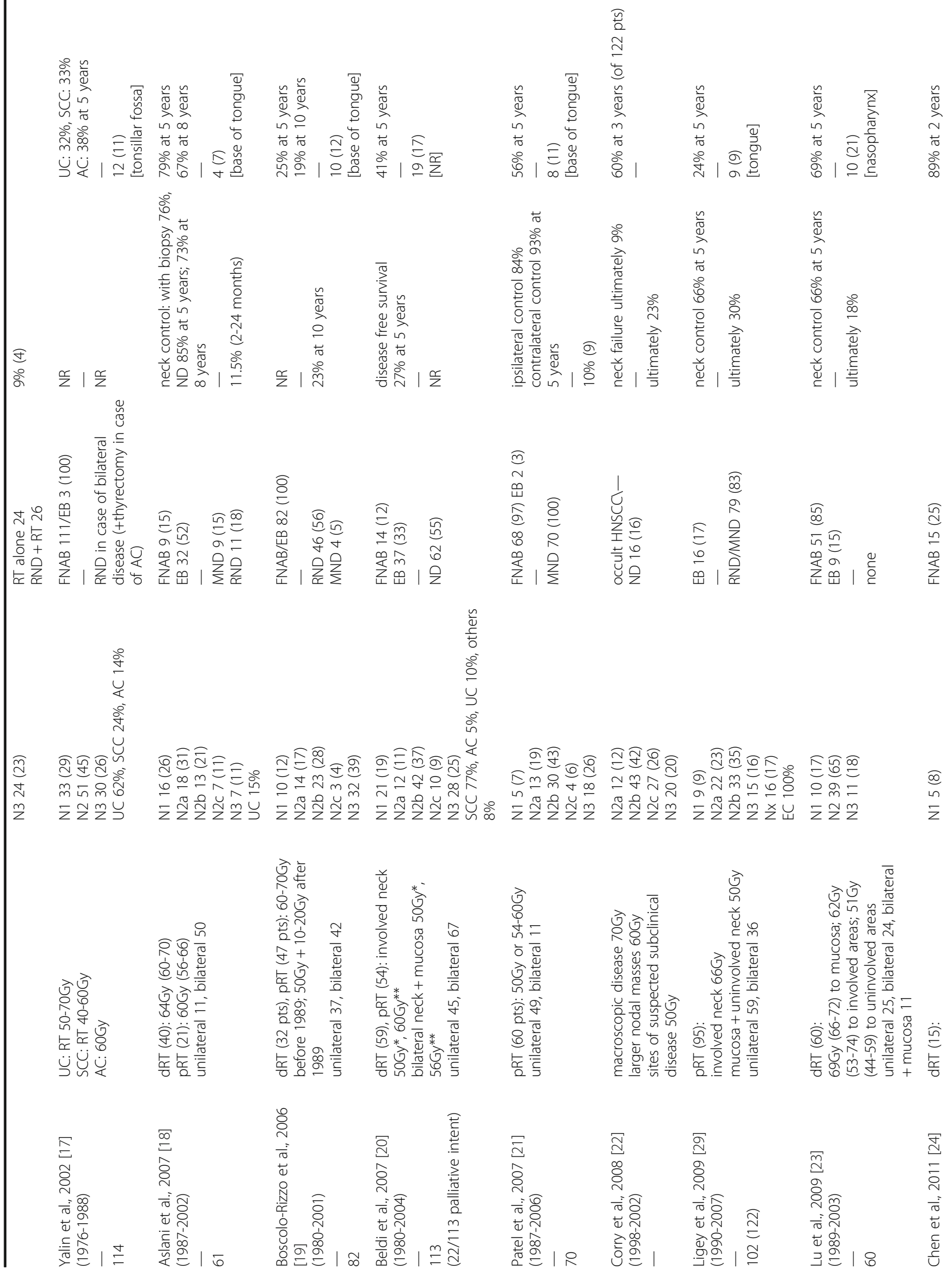




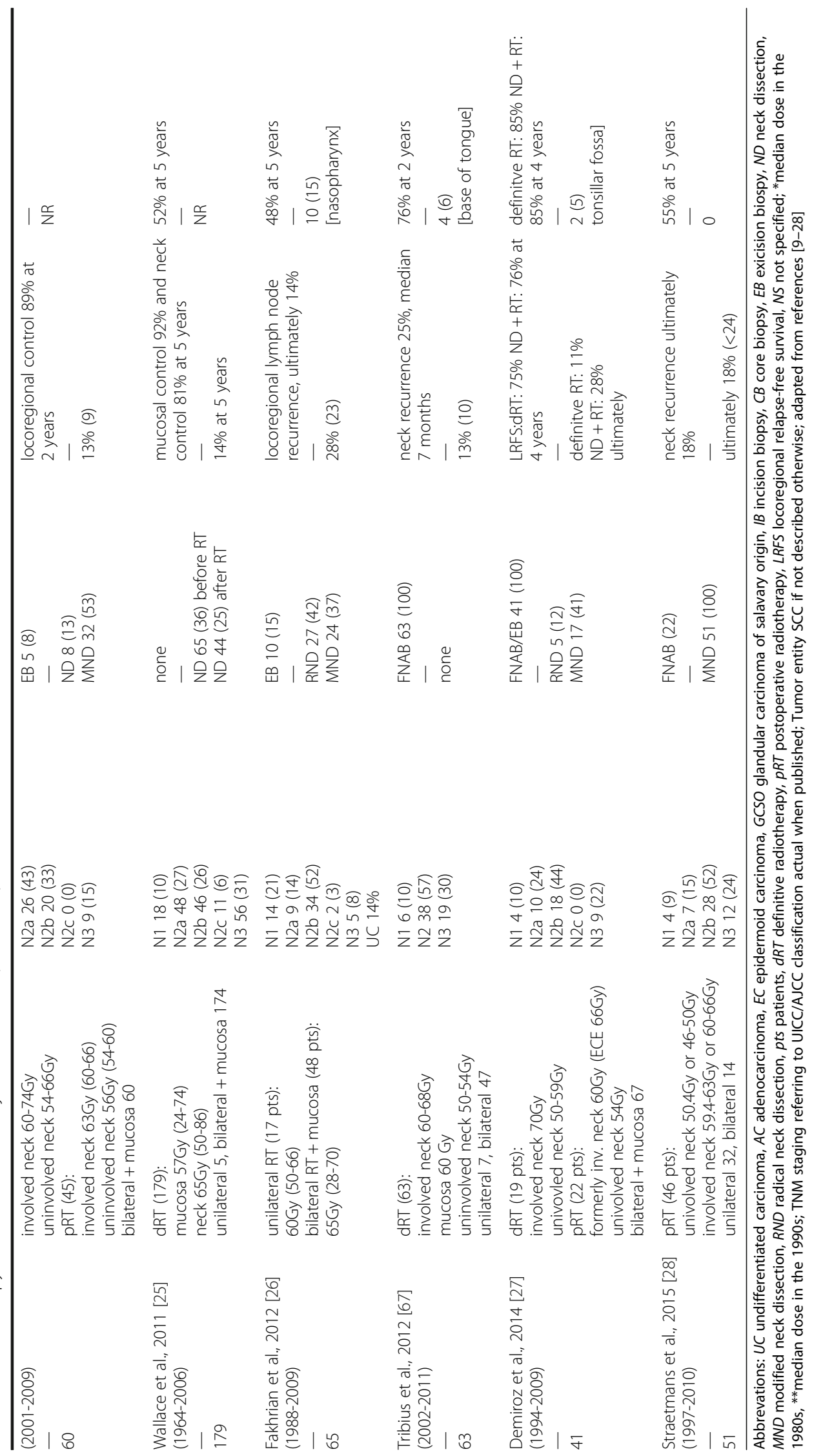


microscopic disease in the neck (R1) [87, 88]. In cases without these risk factors postoperative RT could be considered.

\section{How should advanced-stage neck disease be treated?}

In advanced-stage neck disease (N2b-N3) a multimodal approach consisting of surgery and RT with or witout CTx is most common and seems to provide superior results regarding survival when compared to singlemodality treatment $[15,18,23]$. This is true for the combination of surgery and RT compared to RT alone [25, $27,89]$, as well as for surgery and RT compared to surgery alone, at least regarding the subsequent emergence of a primary tumor [15]. In cases of an unresectable bulk or unambiguously anticipated ECE/incomplete resection, primary chemoradiotherapy (CRT) is the treatment of choice in order to avoid excess toxicity from surgery and postoperative chemoradiotherapy. In most of the retrospective studies above, early-stage disease (1 small node involved) was surgically treated and unresectable masses primary irradiated, which could have biased the results and makes data interpretation difficult. However, due to the lack of prospective data, many crucial questions regarding the optimal radiotherapeutical treatment remain: uni- versus bilateral neck treatment, mucosal irradiation and the use of concomitant chemotherapy.

\section{Which volumes should be irradiated?}

In 2001, Nieder et al. [90] reviewed the management of HNCUP and reported results of various groups regarding ipsilateral versus mucosal and bilateral irradiation. Some results showed decreased tumor control and survival for ipsilateral therapy, while others failed to show any significant differences in outcomes between sole ipsilateral RT and comprehensive treatment of both neck sides and mucosa. When disease control was examined, there was no evidence supporting extended volume treatment over ipsilateral RT. The authors recommended a randomized trial between both options, but a similar trial was never accomplished: a prospective randomized trial (EORTC-24001-22005) starting in 2002 to compare ipsilateral versus bilateral plus mucosal irradiation in HNCUP failed to provide any results, due to very limited patient enrollment. Table 2 demonstrates that most of the larger studies included unilateral as well as bilateral treatment in varying proportions. However, no obvious outcome differences exist between those that treated predominantely unilateral (e.g., Straetmans et al., Patel et al.) and those who preffered a unilateral irradiation (e.g., Wallace et al., Fakhrian et al.), at least regarding overall survival. A recent large meta-analysis revealed no significant differences in 5-year-overall- and disease free survival (OS and DFS) between ipsi- and bilateral RT, but improved locoregional control and lower recurrence rates in favor of bilateral treatment [89]. When considering additional mucosal treatment ("presumed primary tumor"), recurrence rates were significantly lower and DFS better when extended radiation volumes where used, but no benefit for OS could be found and the improved locoregional control was associated with significantly higher severe toxicity [89].

In the current NCCN guidelines [91] no clear statement about the treatment volumes is being made, the approaches found in the literature vary and many of these data originate from the time before the routine use of PET and tonsillectomy. As diagnostic workup became more comprehensive, it could be shown that the numbers of patients developing primary site tumor is lower than indicated in previous literature [15] and also about twofold lower than the risk for nodal recurrence or distant metastases [90]. It seems that metastatic disease in general is nowadays the most common pattern of failure $[92,93]$, so that a possible benefit of a slightly improved locoregional control through extended volume radiotherapy can not be translated in an improved survival [84].

The advent of intensity modulated radiotherapy (IMRT) made the more sophisticated selection of the irradiation volumes essential, as it allows both sparing of organs at risk as well as missing the primary that would had been accidentally treated using older techniques. Previously, HNSCC/HNCUP was treated using a threefield technique including all mucosal sites and both sites of the neck [94], whereas today's standard is intensityIMRT preserving salivatory tissues [95]. The vast majority of the data presented here (Table 2) have been generated with older, non-conformal techniques. However, a possible strategy in modern series treating HNCUP could be the irradiation of selected mucosal sites, e.g., base of tongue for HPV-positive non-smokers or nasopharynx for EBV-positive non-smokers with nonkeratinizing subtypes and/or patients with Asiatic origin. Such approaches have become more common in the IMRTera and the first data are encouraging $[84,96]$.

An overview of the radiation doses and treatment volumes in the greatest series published can be found in Table 2.

\section{Is there a benefit for concomitant chemotherapy?}

The value of adding chemotherapy to RT both in the definitive as in the postoperative setting for treating HNCUP patients remains unclear, despite its common use in many institutions [27, 29, 67, 97, 98]. Cisplatin (e.g., $100 \mathrm{mg} / \mathrm{m}^{2}$, days 1,22 and 43 ) is the agent most frequently used in these cases [24, 99]. Established indications for concomitant chemotherapy in HNSCC are the definitive treatment of locally advanced tumors (e.g., a $\mathrm{cT} 2 \mathrm{cN} 2 \mathrm{~b}$ tumor) or the postoperative treatment of 
Table 3 Concomitant Chemotherapy

\begin{tabular}{|c|c|c|c|}
\hline Study & Concomitant chemotherapy No.(\%) & Locoregional control & Overall survival \\
\hline Yalin, 2002 & $\begin{array}{l}\text { UC, SCC: COP or PCV } \\
\text { AC: PCV } 114(100)\end{array}$ & NR & $\begin{array}{l}\text { UC: } 32 \%, \text { SCC: } 33 \% \\
\text { AC: } 38 \% \text { at } 5 \text { years }\end{array}$ \\
\hline Boscolo-Rizzo, 2006 & Platinum based 9 (11) & NR & $25 \%$ at 5 years, $19 \%$ at 10 years \\
\hline Beldi, 2007 & Platinum based 21 (19) & disease free survival $27 \%$ at 5 years & $41 \%$ at 5 years \\
\hline Corry, 2008 & Platinum based 102 (100) & neck failure ultimately $9 \%$ & $60 \%$ at 3 years \\
\hline Ligey, 2009 & Platinum based 43 (45) & neck control $66 \%$ at 5 years & $24 \%$ at 5 years \\
\hline Lu, 2009 & Platinum based 14 (23) & neck control $66 \%$ at 5 years & $69 \%$ at 5 years \\
\hline Chen, 2011 & Platinum based 32 (53) & locoregional control $89 \%$ at 2 years & $89 \%$ at 2 years \\
\hline Wallace, 2011 & Ctx (drugs NR) 13 (7) & mucosal control $92 \%$ and neck control $81 \%$ at 5 years & $52 \%$ at 5 years \\
\hline Fakhrian, 2012 & $\begin{array}{l}\text { Ctx } 19 \text { (29) } \\
\text { Cis based 10, 5-FU + MMC } 9\end{array}$ & locoregional lymph node recurrence, ultimately $14 \%$ & $48 \%$ at 5 years \\
\hline Tribius, 2012 & Cis $38(60)$ & neck recurrence $25 \%$, median 7 months & $76 \%$ at 2 years \\
\hline Demiroz, 2013 & Ctx, 4 regimes $25(61)$ & $\begin{array}{l}\text { LRFS: dRT: } 75 \% \\
\text { ND + RT: } 76 \% \text { at } 4 \text { years }\end{array}$ & $\begin{array}{l}\text { definitve RT: } 85 \% \\
\text { ND + RT: } 85 \% \text { at } 4 \text { years }\end{array}$ \\
\hline Straetmans, 2014 & Carbo 8 (16) & neck recurrence ultimately $18 \%$ & $55 \%$ at 5 years \\
\hline
\end{tabular}

Abbrevations: SCC squamous cell carcinoma, UC undifferentiated carcinoma, AC adenocarcinoma, EC epidermoid carcinoma, Ctx chemotherapy, Cis cisplatin, 5FU 5-flurouracil, MMC mitomycin C, Carbo carboplatin, COP cyclophosphamide, vincristine, prednisolone, $P C V$ cisplatin, cyclophosphamide, vincristine [10, 11, 53, 76, 77, 79-85]

high-risk tumors (e.g., a pT1pN3b tumor: extracapsular extension). Implementing chemotherapy for a HNCUP with one or more involved nodes after neck dissection would assume that it has a similar prognosis with such cases. This does not seem justified, since a cT1 tumor (in this case not detected, therefore CUP) generally has an excellent prognosis with $\mathrm{RT}$ alone $[84,100]$. A recent study by Hosni et al. revealed an almost identical prognosis for patients with HNCUP and those with T1 baseof-tongue carcinoma [101]. These data would imply that both diseases may be treated the same way, i.e., without the use of chemotherapy. In a retrospective analysis examining the effect of concomitant cisplatin, involving 60 HNCUP-patients, no clear advantage could be found for the addition of chemotherapy and severe toxicities (grade 3+) occurred significantly more often [24]. Furthermore, in the era of HPV/p16 stratification a deescalation of treatment and an alternative staging for positive tumors are already under discussion because of the distinct improved outcomes of this collective [102, 103]. The current paradigm for the indications for postoperative chemo-irradiation (R1, pN3b) originates from the pre-HPV-stratification era $[104,105]$. Keller et al. [70] have conducted an analysis of clinicopathological data, including p16 and extracapsular extension (ECE), in HNCUP and could demonstrate a very similar prognosis in patients with or without ECE, even without chemotherapy, but the patient numbers in this analysis where very limited and so no safe conclusions can be drawn. A treatment-deescalation for HPV/p16 nonsmokers could be imaginable, either through omitting chemotherapy or even by using chemotherapy in order to reduce RT-dose, following the paradigm of current HNSCC trials [102]. Table 3 shows the largest published studies implementing chemotherapy and the agents used in each case [10, 11, 53, 76, 77, 79-85].

\section{Treatment algorithm}

Based on the above considerations we tried to summarize the existing experience and develop a treatment proposal for further evaluation in a prospective mode (Fig. 2).

\section{Conclusions}

To the best of our knowledge, no prospective phase III trial investigating treatment optimization for HNCUP currently exists. Treatment of cervical cancer of unknown primary remains a diagnostic and therapeutical

\section{Neck dissection}

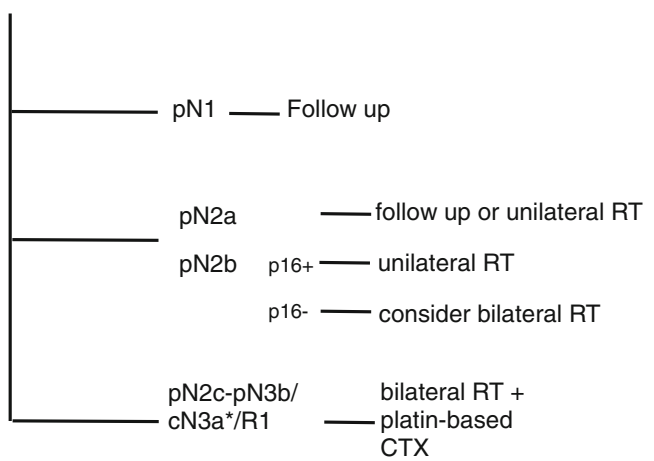

Fig. 2 HNCUP therapy algorithm. RT: radiotherapy, CTX: chemotherapy, *inoperable 
challenge. Several improvements in instrument-based and pathological diagnostics have led to better understanding of this rare disease and less common missing of an undetected primary tumor. Multimodality treatment seems to provide superior results, especially for N2bN3b cases. Until today, there is no unambiguous evidence of a survival benefit through treatment intensification with extended radiotherapy volumes and/or the implementation of concurrent chemotherapy. These questions can only be answered with the help of large prospective trials. Novel molecular parameters like the HPV-status will help stratifying patients for such trials and allow more valid results.

\section{Abbreviations \\ CRT: Chemoradiotherapy; CT: Computer tomography; CTx: Chemotherapy; CUP: Cancer of unknown primary; DFS: Disease free survival; EBV: Epstein-Barr virus; ECE: Extracapsular extention; FDG-PET: 18F-fluoro2-deoxyglucose positron emission tomography; FNAB: Fine-needle aspiration biopsy; HNCUP: CUP in the head and neck region; HNSCC: Head and neck squamous cell carcinoma; HPV: Human papilloma virus; IHC: Immunohistochemistry; IMRT: Intensity modulated radiotherapy; MRI: Magnet resonance imaging; NCCN: National Comprehensive Cancer Network; NPC: Nasopharyngeal carcinoma; OS: Overall survival; P-UADT: Panendoscopy of the upper aerodigestive tract; qRT-PCR: quantitative reverse transcriptase-PCR; RT: Radiotherapy; SCC: Squamous cell carcinoma; UICC: Union Internationale Contre le Cancer}

\section{Acknowledgements}

Not applicable

\section{Funding}

No funding was needed in the design of the study and collection, analysis, and interpretation of data

\section{Availability of data and materials}

Data sharing is not applicable to this article as no datasets were generated or analysed during the current study.

\section{Authors' contributions}

JM, AT, SG, CR and PB analyzed and interpreted the data. All authors read and approved the final manuscript

\section{Competing interests}

The authors declare that they have no competing interests.

\section{Consent for publication}

Not applicable (the manuscript does not contain any individual persons data).

\section{Ethics approval and consent to participate}

As the manuscript does not report on or involve the use of any animal or human data or tissue, ethics approval and consent are not applicable to this submission.

\section{Publisher's Note}

Springer Nature remains neutral with regard to jurisdictional claims in published maps and institutional affiliations.

\footnotetext{
Author details

'Department of Radiation Oncology, University Hospital, Johann Wolfgang Goethe University, Theodor Stern Kai 7, 60590 Frankfurt, Germany. ${ }^{2}$ Department of Otolaryngology and Head and Neck Surgery, Johann Wolfgang Goethe University, Frankfurt, Germany. ${ }^{3}$ Department of Maxillofacial Surgery, Johann Wolfgang Goethe University, Frankfurt, Germany. ${ }^{4}$ German Cancer Research Center (DKFZ), Heidelberg, Germany. ${ }^{5}$ German Cancer Consortium (DKTK), Frankfurt, Germany.
}

Received: 27 January 2017 Accepted: 3 May 2017

Published online: 10 May 2017

\section{References}

1. Califano J, et al. Unknown primary head and neck squamous cell carcinoma: molecular identification of the site of origin. J Natl Cancer Inst. 1999;91(7): 599-604. doi:10.1093/jnci/91.7.599.

2. Pavlidis $\mathrm{N}$, et al. Diagnostic and therapeutic management of cancer of an unknown primary. Eur J Cancer. 2003;39(14):1990-2005. http://dx.doi.org/10. 1016/50959-8049(03)00547-1.

3. Ries $L A G$, Eisner MP, Kosary $C L$, et al. SEER cancer statistics review. Bethesda: National Cancer Institute; 1999.

4. Levi F, et al. Epidemiology of unknown primary tumours. Eur J Cancer. 2002 38(13):1810-2. http://dx.doi.org/10.1016/S0959-8049(02)00135-1.

5. van de Wouw AJ, et al. Epidemiology of unknown primary tumours: incidence and population-based survival of 1285 patients in Southeast Netherlands, 1984-1992. Eur J Cancer. 2002;38(3):409-13. http://dx.doi.org/ 10.1016/S0959-8049(01)00378-1.

6. Strojan $\mathrm{P}$, et al. Combined surgery and postoperative radiotherapy for cervical lymph node metastases from an unknown primary tumour. Radiother Oncol. 1998;49(1):33-40. http://dx.doi.org/10.1016/S01678140(98)00082-6.

7. Regelink $\mathrm{G}$, et al. Detection of unknown primary tumours and distant metastases in patients with cervical metastases: value of FDG-PET versus conventional modalities. Eur J Nucl Med Mol Imaging. 2002;29(8):1024-30. doi:10.1007/s00259-002-0819-0.

8. Issing WJ, et al. Diagnosis and management of carcinoma of unknown primary in the head and neck. Eur Arch Otorhinolaryngol. 2003;260(8):43643. doi:10.1007/s00405-003-0585-z.

9. Bataini JP, et al. Treatment of metastatic neck nodes secondary to an occult epidermoid carcinoma of the head and neck. Laryngoscope. 1987;97(9): 1080-4. doi:10.1288/00005537-198709000-00015.

10. Jesse RH, et al. Cervical lymph node metastasis: unknown primary cancer. Cancer. 1973;31(4):854-9. doi:10.1002/1097-0142(197304)31:4<854::AIDCNCR2820310414>3.0.CO;2-D.

11. Weir $L$, et al. Radiation treatment of cervical lymph node metastases from an unknown primary: an analysis of outcome by treatment volume and other prognostic factors. Radiother Oncol. 1995;35(3):206-11. http://dx.doi. org/10.1016/0167-8140(95)01559-Y.

12. Reddy SP, et al. Metastatic carcinoma in the cervical lymph nodes from an unknown primary site: results of bilateral neck plus mucosal irradiation vs. ipsilateral neck irradiation. Int J Radiat Oncol Biol Phys. 1997;37(4):797-802. http://dx.doi.org/10.1016/S0360-3016(97)00025-4.

13. Colletier PJ, et al. Postoperative radiation for squamous cell carcinoma metastatic to cervical lymph nodes from an unknown primary site: outcomes and patterns of failure. Head Neck. 1998;20(8):674-81. doi:10. 1002/(SICI) 1097-0347(199812)20:8<674::AID-HED3>3.0.CO;2-H.

14. Fernandez JA, et al. Metastatic squamous cell carcinoma in cervical lymph nodes from an unknown primary tumour: prognostic factors. Clin Otolaryngol Allied Sci. 1998;23(2):158-63. doi:10.1046/j.1365-2273.1998. 00122.x.

15. Grau C, et al. Cervical lymph node metastases from unknown primary tumours. Results from a national survey by the Danish Society for Head and Neck Oncology. Radiother Oncol. 2000;55(2):121-9. http://dx.doi.org/10. 1016/S0167-8140(00)00172-9.

16. Iganej S, et al. Metastatic squamous cell carcinoma of the neck from an unknown primary: management options and patterns of relapse. Head Neck. 2002;24(3):236-46.

17. Yalin Y, et al. Management and outcome of cervical lymph node metastases of unknown primary sites: a retrospective study. Br J Oral Maxillofac Surg. 2002;40(6):484-7. http://dx.doi.org/10.1016/\$0266435602002449.

18. Aslani $\mathrm{M}$, et al. Metastatic carcinoma to the cervical nodes from an unknown head and neck primary site: Is there a need for neck dissection? Head Neck. 2007;29(6):585-90. doi:10.1002/hed.20581.

19. Boscolo-Rizzo P, et al. Cervical lymph node metastases from occult squamous cell carcinoma: analysis of 82 cases. ORL J Otorhinolaryngol Relat Spec. 2006;68(4):189-94. doi:10.1159/000091394.

20. Beldi $\mathrm{D}$, et al. Role of radiotherapy in the treatment of cervical lymph node metastases from an unknown primary site: retrospective analysis of 113 patients. Int J Radiat Oncol Biol Phys. 2007;69(4):1051-8. doi:10.1016/j.j.jrobp. 2007.04.039 
21. Patel RS, et al. Squamous cell carcinoma from an unknown head and neck primary site: a "selective treatment" approach. Arch Otolaryngol Head Neck Surg. 2007;133(12):1282-7. doi:10.1001/archotol.133.12.1282.

22. Corry J, et al. The role of PET-CT in the management of patients with advanced cancer of the head and neck. Curr Oncol Rep. 2008;10(2):149-55. doi:10.1007/s11912-008-0023-x.

23. Lu X, et al. Squamous cell carcinoma metastatic to cervical lymph nodes from an unknown primary site: the impact of radiotherapy. Tumori. 2009; 95(2):185-90. doi:10.1700/422.5007.

24. Chen AM, et al. Radiation therapy in the management of head-and-neck cancer of unknown primary origin: how does the addition of concurrent chemotherapy affect the therapeutic ratio? Int J Radiat Oncol Biol Phys. 2011;81(2):346-52. doi:10.1016/j.jirobp.2010.06.031.

25. Wallace $A$, et al. Head and neck squamous cell carcinoma from an unknown primary site. Am J Otolaryngol. 2011;32(4):286-90. doi:10.1016/j.amjoto.2010. 05.004.

26. Fakhrian $\mathrm{K}$, et al. Radio(chemo)therapy in the management of squamous cell carcinoma of cervical lymph nodes from an unknown primary site. A retrospective analysis. Strahlenther Onkol. 2012;188(1):56-61. doi:10.1007/ s00066-011-0017-8

27. Demiroz C, et al. Head and neck squamous cell carcinoma of unknown primary: neck dissection and radiotherapy or definitive radiotherapy. Head Neck. 2014;36(11):1589-95. doi:10.1002/hed.23479.

28. Straetmans J, et al. Management of neck metastases of unknown primary origin united in two European centers. Eur Arch Otorhinolaryngol. 2015; 272(1):195-205. doi:10.1007/s00405-014-2934-5.

29. Ligey $\mathrm{A}$, et al. Impact of target volumes and radiation technique on locoregional control and survival for patients with unilateral cervical lymph node metastases from an unknown primary. Radiother Oncol. 2009;93(3): 483-7. doi:10.1016/j.radonc.2009.08.027.

30. Haas I, et al. Diagnostic strategies in cervical carcinoma of an unknown primary (CUP). Eur Arch Otorhinolaryngol. 2002;259(6):325-33. doi:10.1007/ s00405-002-0470-1

31. Shukla P, et al. Metastatic squamous cell carcinoma neck with occult primary: a retrospective analysis. Indian J Med Paediatr Oncol. 2009;30(4): 124-30. doi:10.4103/0971-5851.65334.

32. Mondin V, et al. A survey of metastatic central nervous system tumors to cervical lymph nodes. Eur Arch Otorhinolaryngol. 2010;267(11):1657-66. doi: 10.1007/s00405-010-1357-1.

33. Shah JP. Patterns of cervical lymph node metastasis from squamous carcinomas of the upper aerodigestive tract. Am J Surg. 1990;160(4):405-9.

34. Nguyen C, et al. Metastatic squamous cell carcinoma to cervical lymph nodes from unknown primary mucosal sites. Head Neck. 1994;16(1):58-63. doi:10.1002/hed.2880160112.

35. D'Souza G, et al. Moderate predictive value of demographic and behavioral characteristics for a diagnosis of HPV16-positive and HPV16-negative head and neck cancer. Oral Oncol. 2010;46(2):100-4. doi:10.1016/j.oraloncology. 2009.11.004.

36. Kreimer AR, et al. Human papillomavirus types in head and neck squamous cell carcinomas worldwide: a systematic review. Cancer Epidemiol Biomarkers Prev. 2005;14(2):467-75. doi:10.1158/1055-9965.EPI-04-0551.

37. Gillison $\mathrm{ML}$, et al. Distinct risk factor profiles for human papillomavirus type 16-positive and human papillomavirus type 16-negative head and neck cancers. J Natl Cancer Inst. 2008;100(6):407-20. doi:10.1093/jnci/djn025.

38. Fakhry $\mathrm{C}$, et al. Improved survival of patients with human papillomaviruspositive head and neck squamous cell carcinoma in a prospective clinical trial. J Natl Cancer Inst. 2008;100(4):261-9. doi:10.1093/jnci/djn011.

39. Coster JR, et al. Cervical nodal metastasis of squamous cell carcinoma of unknown origin: indications for withholding radiation therapy. Int J Radiat Oncol Biol Phys. 1992;23(4):743-9. http://dx.doi.org/10.1016/03603016(92)90647-Z.

40. Glynne-Jones RG, et al. Metastatic carcinoma in the cervical lymph nodes from an occult primary: a conservative approach to the role of radiotherapy. Int J Radiat Oncol Biol Phys. 1990;18(2):289-94. http://dx.doi.org/10.1016/ 0360-3016(90)90091-W

41. Golfinopoulos V, et al. Comparative survival with diverse chemotherapy regimens for cancer of unknown primary site: multiple-treatments metaanalysis. Cancer Treat Rev. 2009;35(7):570-3. doi:10.1016/j.ctrv.2009.05.005.

42. Pentheroudakis $\mathrm{G}$, et al. Cancer of unknown primary site: missing primary or missing biology? Oncologist. 2007;12(4):418-25. doi:10.1634/theoncologist. 12-4-418.
43. Strojan $\mathrm{P}$, et al. Contemporary management of lymph node metastases from an unknown primary to the neck: I. A review of diagnostic approaches. Head Neck. 2013;35(1):123-32. doi:10.1002/hed.21898.

44. Shaha AR, Rinaldo A, Ferlito A. Metastatic squamous carcinoma in the neck from an occult primary. In: Jackler RK, Ferlito A, Silver CE, editors. Neck dissection. Management of regional disease in head and neck cancer. San Diego: Plural Publishing; 2010. p. 307-17.

45. Lester J, et al. Random periareolar fine-needle aspiration: the new pap smear of the breast? J Adv Pract Oncol. 2012;3(6):411-4.

46. Oien KA. Pathologic evaluation of unknown primary cancer. Semin Oncol. 2009;36(1):8-37. doi:10.1053/j.seminoncol.2008.10.009.

47. Waltonen JD, et al. Metastatic carcinoma of the neck of unknown primary origin: evolution and efficacy of the modern workup. Arch Otolaryngol Head Neck Surg. 2009;135(10):1024-9. doi:10.1001/archoto.2009.145.

48. Muraki AS, et al. Metastatic cervical adenopathy from tumors of unknown origin: the role of CT. Radiology. 1984;152(3):749-53. doi:10.1148/radiology. 152.3.6463256.

49. Freudenberg LS, et al. Dual modality of 18 F-fluorodeoxyglucose-positron emission tomography/computed tomography in patients with cervical carcinoma of unknown primary. Med Princ Pract. 2005;14(3):155-60. doi:10. $1159 / 000084632$

50. Cianchetti $M$, et al. Diagnostic evaluation of squamous cell carcinoma metastatic to cervical lymph nodes from an unknown head and neck primary site. Laryngoscope. 2009;119(12):2348-54. doi:10.1002/lary.20638.

51. Zhuang SM, et al. Management of lymph node metastases from an unknown primary site to the head and neck (Review). Mol Clin Oncol. 2014; 2(6):917-22. doi:10.3892/mco.2014.361.

52. Johansen J, et al. Prospective study of 18FDG-PET in the detection and management of patients with lymph node metastases to the neck from an unknown primary tumor. Results from the DAHANCA-13 study. Head Neck. 2008;30(4):471-8. doi:10.1002/hed.20734

53. Seve $\mathrm{P}$, et al. The role of 2-deoxy-2-[F-18]fluoro-D-glucose positron emission tomography in disseminated carcinoma of unknown primary site. Cancer. 2007:109(2):292-9. doi:10.1002/cncr.22410.

54. Rusthoven KE, et al. The role of fluorodeoxyglucose positron emission tomography in cervical lymph node metastases from an unknown primary tumor. Cancer. 2004;101(11):2641-9. doi:10.1002/cncr.20687.

55. Al-Ibraheem A, et al. Clinical applications of FDG PET and PET/CT in head and neck cancer. J Oncol. 2009;2009:208725. doi:10.1155/2009/208725.

56. Kwee TC, et al. Combined FDG-PET/CT for the detection of unknown primary tumors: systematic review and meta-analysis. Eur Radiol. 2009;19(3): 731-44. doi:10.1007/s00330-008-1194-4

57. de Bree R. The real additional value of FDG-PET in detecting the occult primary tumour in patients with cervical lymph node metastases of unknown primary tumour. Eur Arch Otorhinolaryngol. 2010;267(11):1653-5. doi:10.1007/s00405-010-1372-2.

58. Jones AS, et al. Squamous carcinoma presenting as an enlarged cervical lymph node. Cancer. 1993;72(5):1756-61. doi:10.1002/10970142(19930901)72:5<1756::AID-CNCR2820720540>3.0.CO;2-5.

59. Ang KK, et al. Human papillomavirus and survival of patients with oropharyngeal cancer. N Engl J Med. 2010;363(1):24-35. doi:10.1056/ NEJMoa0912217.

60. Rischin D, et al. Prognostic significance of p16INK4A and human papillomavirus in patients with oropharyngeal cancer treated on TROG 02 02 phase III trial. J Clin Oncol. 2010;28(27):4142-8. doi:10.1200/JCO.2010.29. 2904.

61. Lingen MW, et al. Low etiologic fraction for high-risk human papillomavirus in oral cavity squamous cell carcinomas. Oral Oncol. 2013;49(1):1-8. doi:10. 1016/j.oraloncology.2012.07.002.

62. Shi W, et al. Comparative prognostic value of HPV16 E6 mRNA compared with in situ hybridization for human oropharyngeal squamous carcinoma. J Clin Oncol. 2009;27(36):6213-21. doi:10.1200/JCO.2009.23.1670.

63. Jordan RC, et al. Validation of methods for oropharyngeal cancer HPV status determination in US cooperative group trials. Am J Surg Pathol. 2012;36(7): 945-54. doi:10.1097/PAS.0b013e318253a2d1

64. Rietbergen MM, et al. Human papillomavirus detection and comorbidity: critical issues in selection of patients with oropharyngeal cancer for treatment De-escalation trials. Ann Oncol. 2013;24(11):2740-5. doi:10.1093/ annonc/mdt319.

65. Chung $\mathrm{CH}_{\text {, et }}$ al. p16 protein expression and human papillomavirus status as prognostic biomarkers of nonoropharyngeal head and neck squamous 
cell carcinoma. J Clin Oncol. 2014;32(35):3930-8. doi:10.1200/JCO.2013.54. 5228.

66. Dixon PR, et al. Impact of p16 expression, nodal status, and smoking on oncologic outcomes of patients with head and neck unknown primary squamous cell carcinoma. Head Neck. 2016;38(9):1347-53. doi:10.1002/hed. 24441.

67. Tribius S, et al. HPV status in patients with head and neck of carcinoma of unknown primary site: HPV, tobacco smoking, and outcome. Oral Oncol. 2012;48(11):1178-84. doi:10.1016/j.oraloncology.2012.05.022.

68. Ragin CC, et al. Survival of squamous cell carcinoma of the head and neck in relation to human papillomavirus infection: review and meta-analysis. Int J Cancer. 2007;121(8):1813-20. doi:10.1002/ijc.22851.

69. Vent J, et al. p16 expression in carcinoma of unknown primary: diagnostic indicator and prognostic marker. Head Neck. 2013;35(11):1521-6. doi:10. 1002/hed.23190.

70. Keller LM, et al. p16 status, pathologic and clinical characteristics, biomolecular signature, and long-term outcomes in head and neck squamous cell carcinomas of unknown primary. Head Neck. 2014;36(12): 1677-84. doi:10.1002/hed.23514.

71. Duffy MJ, et al. p53 as a target for the treatment of cancer. Cancer Treat Rev. 2014;40(10):1153-60. doi:10.1016/j.ctrv.2014.10.004

72. Poeta ML, et al. TP53 mutations and survival in squamous-cell carcinoma of the head and neck. N Engl J Med. 2007;357(25):2552-61. doi:10.1056/ NEJMoa073770.

73. Sivars $L$, et al. Human papillomavirus and p53 expression in cancer of unknown primary in the head and neck region in relation to clinical outcome. Cancer Med. 2014;3(2):376-84. doi:10.1002/cam4.199.

74. Pezzuto F, et al. Update on head and neck cancer: current knowledge on epidemiology, risk factors, molecular features and novel therapies. Oncology. 2015;89(3):125-36. doi:10.1159/000381717.

75. Yoshizaki T, et al. Pathogenic role of Epstein-Barr virus latent membrane protein-1 in the development of nasopharyngeal carcinoma. Cancer Lett. 2013;337(1):1-7. doi:10.1016/j.canlet.2013.05.018.

76. Raab-Traub N. Epstein-Barr virus in the pathogenesis of NPC. Semin Cancer Biol. 2002;12(6):431-41. http://dx.doi.org/10.1016/S1044579X0200086X.

77. Yoshizaki T. Promotion of metastasis in nasopharyngeal carcinoma by Epstein-Barr virus latent membrane protein-1. Histol Histopathol. 2002;17(3): 845-50.

78. Yoshizaki T, et al. Current understanding and management of nasopharyngeal carcinoma. Auris Nasus Larynx. 2012;39(2):137-44. doi:10. 1016/j.anl.2011.02.012.

79. Macdonald MR, et al. Role of Epstein-Barr virus in fine-needle aspirates of metastatic neck nodes in the diagnosis of nasopharyngeal carcinoma. Head Neck. 1995;17(6):487-93.

80. Lei ZX, et al. Detection of Epstein-Barr virus DNA in fine needle aspiration specimen from cervical lymphnodes with polymerase chain reaction. Lin Chuang Er Bi Yan Hou Ke Za Zhi. 2000;14(10):454-5.

81. Yap YY, et al. Epstein-Barr virus DNA detection in the diagnosis of nasopharyngeal carcinoma. Otolaryngol Head Neck Surg. 2007;136(6):98691. doi:10.1016/j.otohns.2006.11.027.

82. Plaza G, et al. Neck lymph node metastases of unknown origin: nasopharyngeal origin and EBV (Epstein-Barr virus). Acta Otorrinolaringol Esp. 1999;50(8):623-9.

83. Strojan $\mathrm{P}$, et al. Contemporary management of lymph node metastases from an unknown primary to the neck: II. a review of therapeutic options. Head Neck. 2013;35(2):286-93. doi:10.1002/hed.21899.

84. Galloway TJ, et al. Management of squamous cancer metastatic to cervical nodes with an unknown primary site. J Clin Oncol. 2015;33(29):3328-37. doi: 10.1200/JCO.2015.61.0063.

85. Suarez C, et al. Neck dissection with or without postoperative radiotherapy in supraglottic carcinomas. Otolaryngol Head Neck Surg. 1993;109(1):3-9.

86. Rodrigo JP, et al. Efficacy of postoperative radiation therapy for squamous cell carcinoma of the head and neck: results of a prospective randomised clinical trial. Acta Otorrinolaringol Esp. 2004;55(9):415-9. doi:10.1016/500016519(04)78546-5.

87. Wang $\mathrm{RC}$, et al. Unknown primary squamous cell carcinoma metastatic to the neck. Arch Otolaryngol Head Neck Surg. 1990;116(12):1388-93. doi:10. 1001/archotol.1990.01870120034004.

88. Fletcher GH, Jesse Jr RH, Lindberg RD, Westbrook KC. Neck nodes. In: Fletcher GH, editor. Textbook of radiotherapy. 3rd ed. Philadelphia: Lea \& Febiger; 1980. p. 249-71.
89. Liu X, et al. Optimization of radiotherapy for neck carcinoma metastasis from unknown primary sites: a meta-analysis. Oncotarget. 2016. doi:10. 18632/oncotarget.12852.

90. Nieder C, et al. Cervical lymph node metastases from occult squamous cell carcinoma: cut down a tree to get an apple? Int J Radiat Oncol Biol Phys. 2001;50(3):727-33. http://dx.doi.org/10.1016/S0360-3016(01)01462-6.

91. NCCN: National Comprehensive Cancer Network. https://www.nccn.org/ professionals/physician_gls/pdf/head-and-neck.pdf Accessed 28 Nov 2016.

92. Gani $\mathrm{C}$, et al. Cervical squamous cell lymph node metastases from an unknown primary site: survival and patterns of recurrence after radiotherapy. Clin Med Insights Oncol. 2013;7:173-80. doi:10.4137/CMO.S12169.

93. Rodel RM, et al. Impact of distant metastasis in patients with cervical lymph node metastases from cancer of an unknown primary site. Ann Otol Rhinol Laryngol. 2009;118(9):662-9. doi:10.1177/000348940911800911.

94. Million RR CN, Mancuso AAL. The unknown primary. In: Cassisi N, Million RR, editors. Management of head and neck cancer: a multidisciplinary approach. 2nd ed. Philadelphia: PA, Lippincott; 1994. p. 311-20.

95. Frank SJ, et al. Intensity-modulated radiotherapy for cervical node squamous cell carcinoma metastases from unknown head-and-neck primary site: M. D. Anderson Cancer Center outcomes and patterns of failure. Int J Radiat Oncol Biol Phys. 2010;78(4):1005-10. doi:10.1016/j.ijrobp.2009.09.006.

96. Mourad WF, et al. Initial experience with oropharynx-targeted radiation therapy for metastatic squamous cell carcinoma of unknown primary of the head and neck. Anticancer Res. 2014;34(1):243-8.

97. Shehadeh NJ, et al. Benefit of postoperative chemoradiotherapy for patients with unknown primary squamous cell carcinoma of the head and neck. Head Neck. 2006;28(12):1090-8. doi:10.1002/hed.20470.

98. Perkins SM, et al. Radiotherapeutic management of cervical lymph node metastases from an unknown primary site. Arch Otolaryngol Head Neck Surg. 2012;138(7):656-61. doi:10.1001/archoto.2012.1110.

99. Klem ML, et al. Intensity-modulated radiotherapy for head and neck cancer of unknown primary: toxicity and preliminary efficacy. Int J Radiat Oncol Biol Phys. 2008;70(4):1100-7. doi:10.1016/j.jijobp.2007.07.2351.

100. Garden AS, et al. Is concurrent chemoradiation the treatment of choice for all patients with Stage III or IV head and neck carcinoma? Cancer. 2004; 100(6):1171-8. doi:10.1002/cncr.20069.

101. Hosni A, et al. Radiotherapy characteristics and outcomes for head and neck carcinoma of unknown primary vs T1 base-of-tongue carcinoma. JAMA Otolaryngol Head Neck Surg. 2016. doi:10.1001/jamaoto.2016.3083.

102. Masterson L, et al. De-escalation treatment protocols for human papillomavirus-associated oropharyngeal squamous cell carcinoma: a systematic review and meta-analysis of current clinical trials. Eur J Cancer. 2014;50(15):2636-48. doi:10.1016/j.ejca.2014.07.001.

103. O'Sullivan B, et al. Development and validation of a staging system for HPVrelated oropharyngeal cancer by the International Collaboration on Oropharyngeal cancer Network for Staging (ICON-S): a multicentre cohort study. Lancet Oncol. 2016;17(4):440-51. doi:10.1016/S1470-2045(15)00560-4.

104. Cooper JS, et al. Postoperative concurrent radiotherapy and chemotherapy for high-risk squamous-cell carcinoma of the head and neck. N Engl J Med. 2004;350(19):1937-44. doi:10.1056/NEJMoa032646.

105. Bernier J, et al. Postoperative irradiation with or without concomitant chemotherapy for locally advanced head and neck cancer. N Engl J Med. 2004;350(19):1945-52. doi:10.1056/NEJMoa032641. 